\title{
COVID 19 - CHALLENGES, ECONOMIC PROBLEMS
}

\author{
MIKHEIL CHIKVILADZE \\ Doctor of Economics, Associate Professor \\ Ivane Javakhishvili Tbilisi State \\ University, Georgia \\ m_chikviladze@mail.ru
}

Abstract. The article is focused on problems in the Georgian economy caused by the new coronavirus epidemic. The current implementation of the state budget of Georgia for 2020 has been analyzed, attention has been drawn to the difficulties of both tax and total revenue performance. The main focus is on the effectiveness of business support measures and the transparency and legality of spending money. Measures have been taken on the operative elaboration and implementation of the relevant micro-fiscal policy, in response to the shocks in demand-supply.

Coronavirus has caused many problems in the world economy. The world economy fell by $7.5 \%$ in the United States on March 9, which has not happened on Wall Street since 2008 global economic crisis. Of course, the influence of Coronavirus on the Georgia's economy is severe, and the country is trying to deal with it in every possible way. If we analyze the current state budget execution this year, it can be seen that the current state budget for 2020 has been implemented in the first quarter. However, we should not expect the second quarter of the state budget for the same year, as this trend is already visible in April and May. The problems will be reflected in May, both in terms of tax revenues and total revenues as well, which is expected to double the state budget deficit of $2.5 \%$, which means a complete failure of the two quarters.

It can be said that the shock of the economy that accompanies coronavirus is quite strange and it affects to the supply and demand at the same time.

We see negative risks on the supply side, which is reflected in the high cost of doing business, the demand will decrease as for increased uncertainty, tighter security measures and restrictions on free movement, as a result, the ability of consumers to spend their own money will be reduced.

Experience has shown that $1 / 3$ of the economic losses will be caused by direct losses: job cuts, quarantine and etc. $2 / 3$ of economic losses will be indirect - which will be related to the decrease in consumer confidence, changes in the behavior of business entities, as well as the tightening of access to credit resources. It can be said that the global financial market is now more stable than it was before the 2008 crisis, but the main challenge in the current situation is still to manage uncertainty. In such a situation, it is inevitable that the funds from the budget will be prioritized for health care, so that the health care of the citizens not to be hindered. It seems inevitable for us to resolve the demand - to develop appropriate microfiscal policies in response to the shocks in supply.

It is welcome that the European Investment Bank supports Georgia in the fight against the new coronavirus, which is to support Georgian business, support the health care system and accelerate the implementation of existing projects.

The World Bank provided \$ 15 billion quick and instant assistance to address the first challenges of COVID-19, this was mainly in the field of health and social care. The World Bank is currently working on three financial assistance packages: 1. To assist the government in responding to health and social protection challenges, 2 . Substantiation will be provided to the state budget to reduce the expected deficit, 3. The World Bank intends to help small and medium-sized businesses to restore jobs and develop the economy, with a particular focus on affected sectors such as tourism, the first phase shall be completed by the end of April, which will be followed consistently. The World Bank has highlighted such an important circumstance as the inevitability of a zoological recession, which will be followed by a slowdown in economic growth, which in the case of Georgia will be $4 \%$ (which is significant to consider). According to the governmentıs decision, 2 billion GEL has been allocated for direct assistance to the economy, in order to provide emergency financial and material assistance to a number of different categories of affected citizens.

Particular attention is paid to operative execution and control of measures envisaged in the concrete plan of economic stimulus and the anti-crisis plan of the government.

KEYWORDS: BUDGET, COVID 19, ACCOUNTS RECEIVABLE, PENSION PROVISION.

For citation: Chikviladze, M., (2020). Covid-19 - Challenges, Economic Problems. Globalization and Business, 10. 70-74. (In Georgian) https://doi.org/10.35945/gb.2020.10.008 


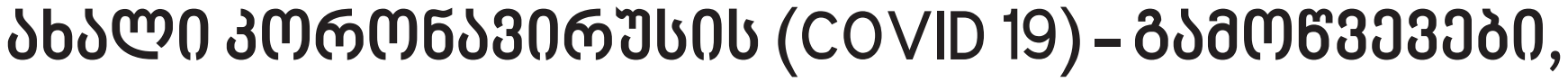

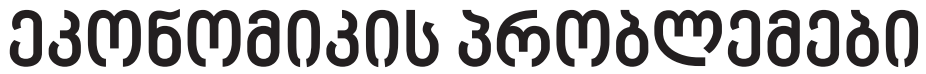

\section{a0bo0ल 60330लsdo}

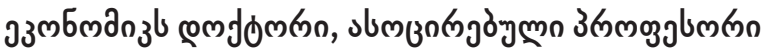

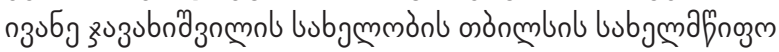

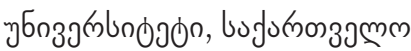 \\ m_chikviladze@mail.ru
}

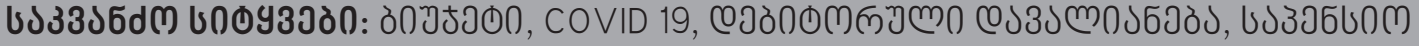

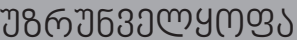

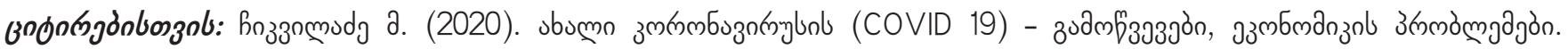

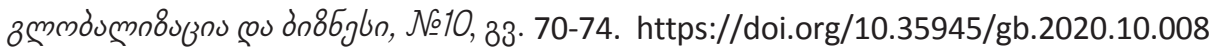

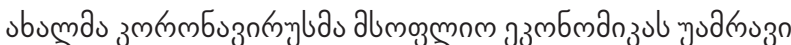

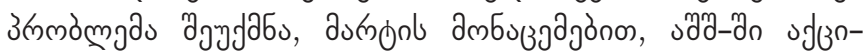

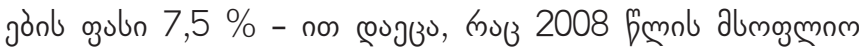

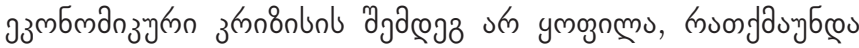

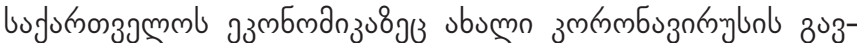

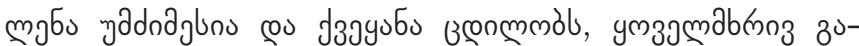

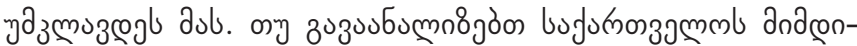

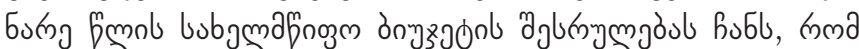

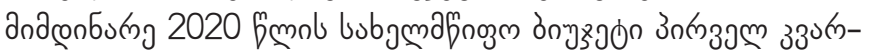

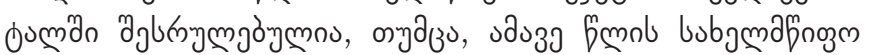

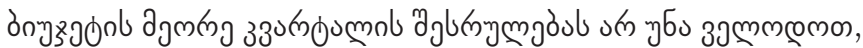

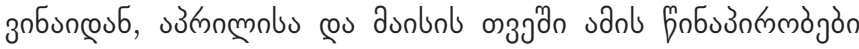

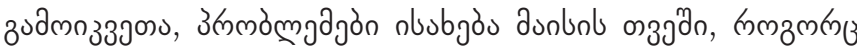

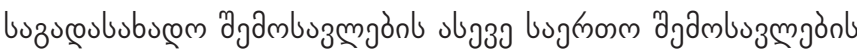

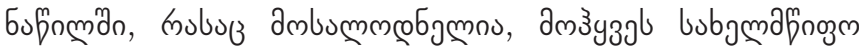

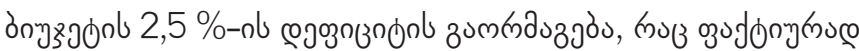

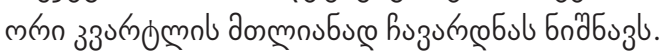

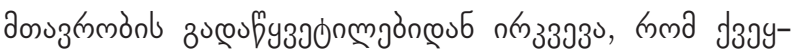

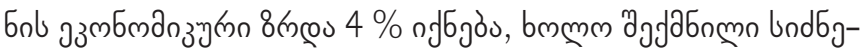

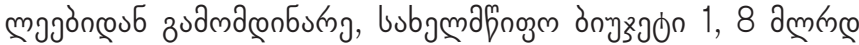

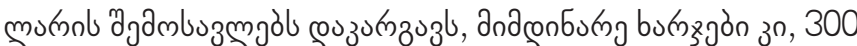
a

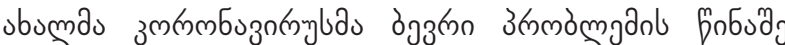

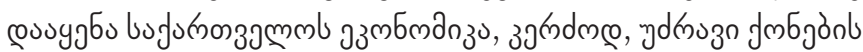

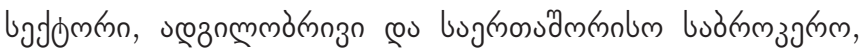

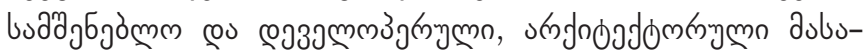

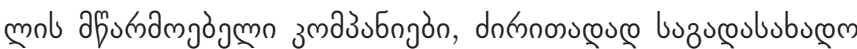

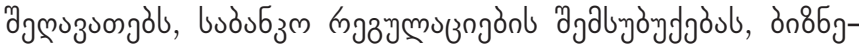

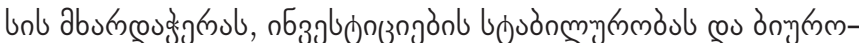

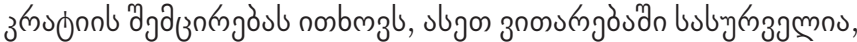

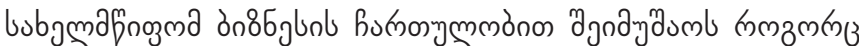

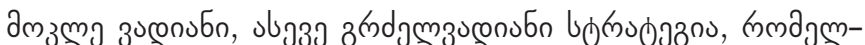

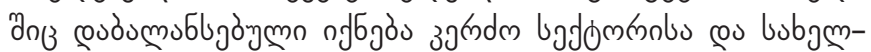

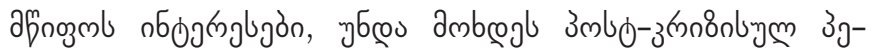

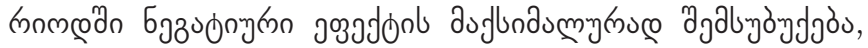

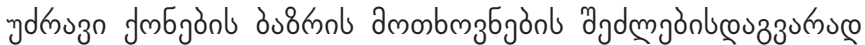
coszas ymoुnm

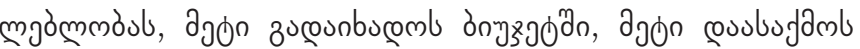

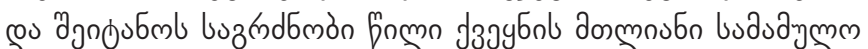

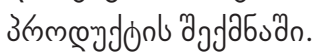

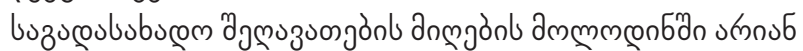

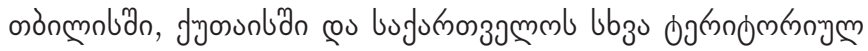

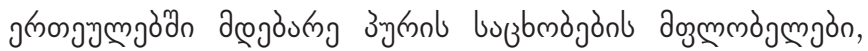

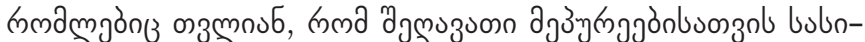

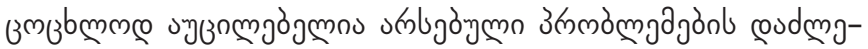

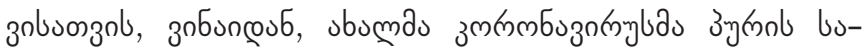

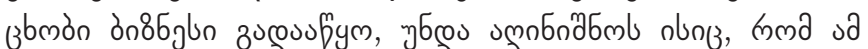

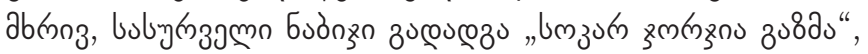

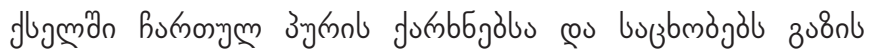

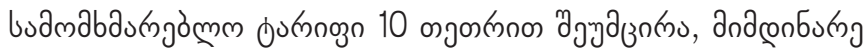

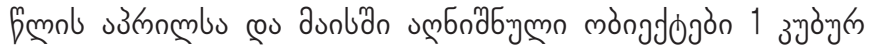

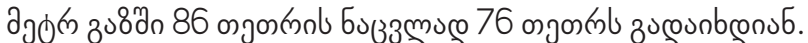

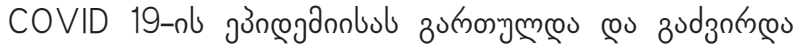

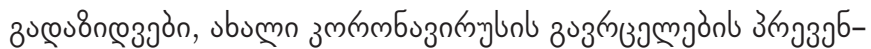

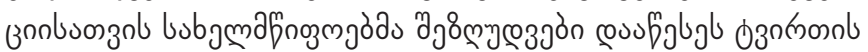

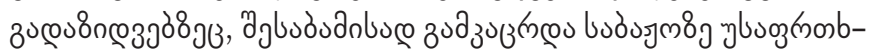

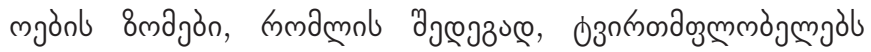

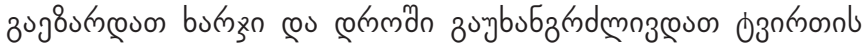

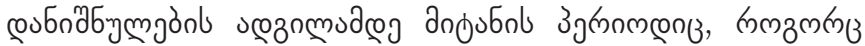

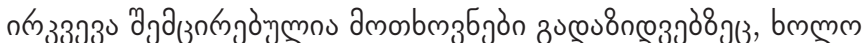

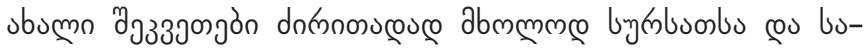

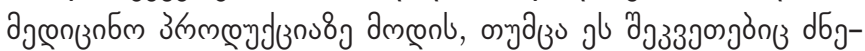




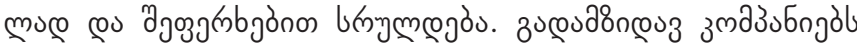

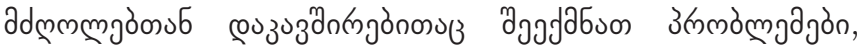
zucou8n@

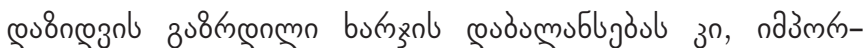

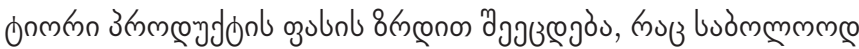

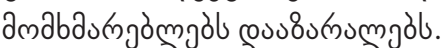

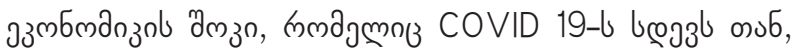

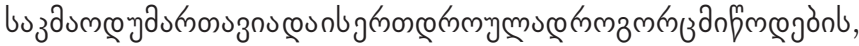

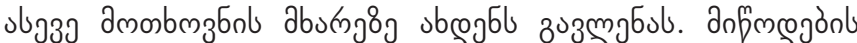

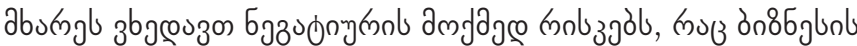

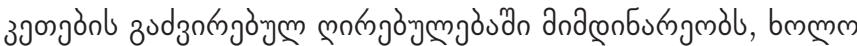

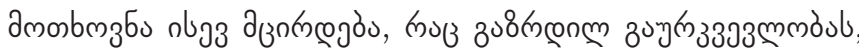

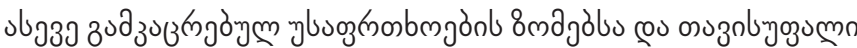

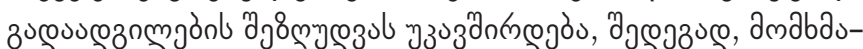

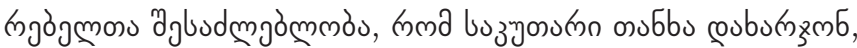

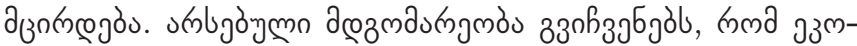

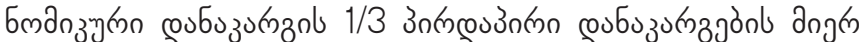

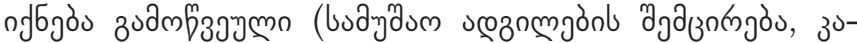

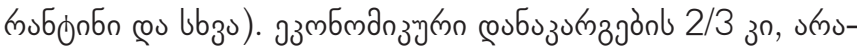

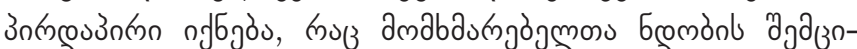

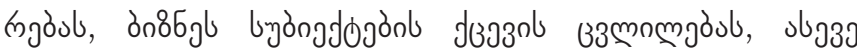

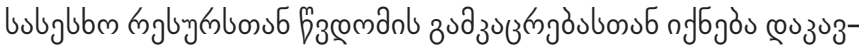

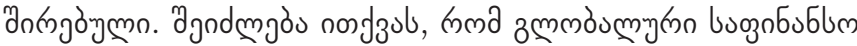

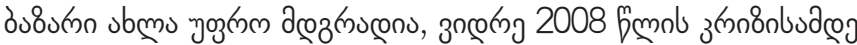

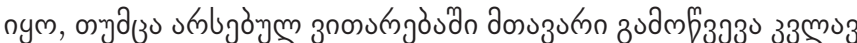

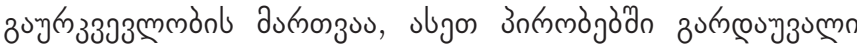

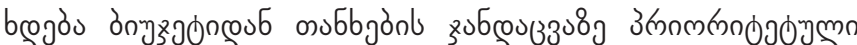

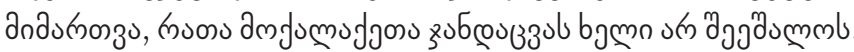

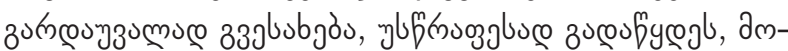

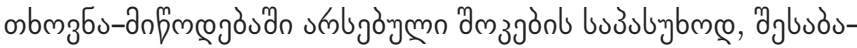

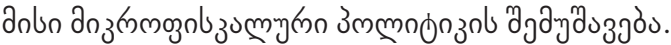

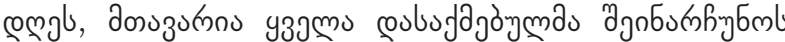

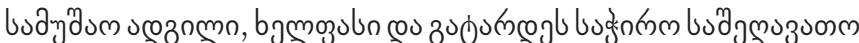

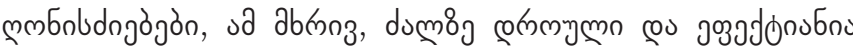

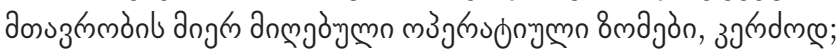

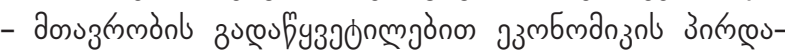

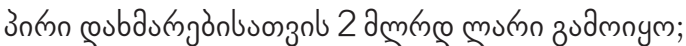

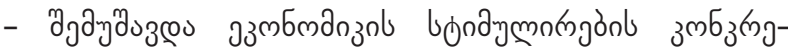

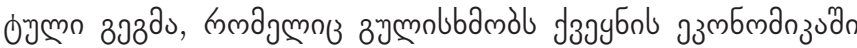

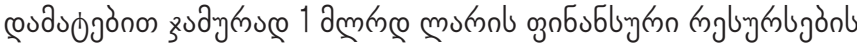
3ohjoul;

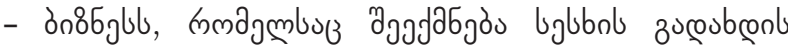

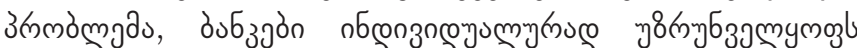

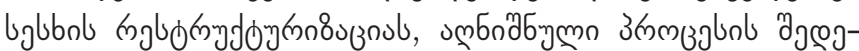

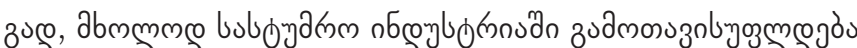

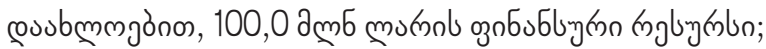

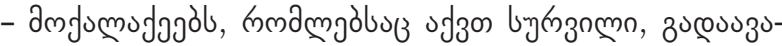

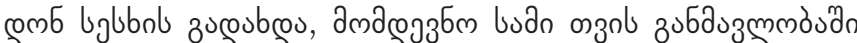

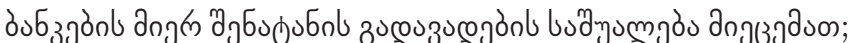

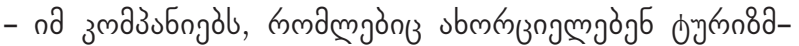

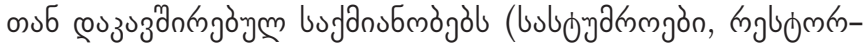

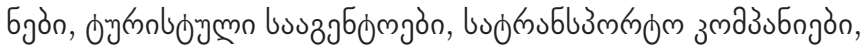

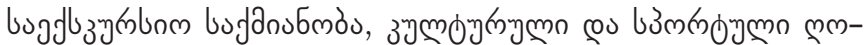

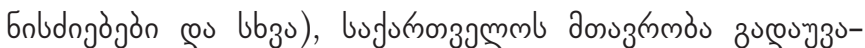

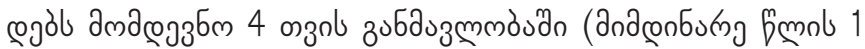

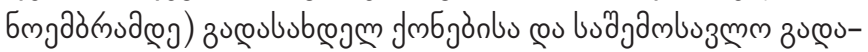

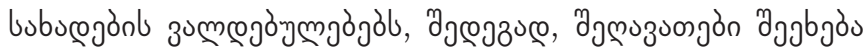

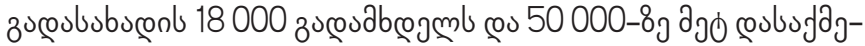

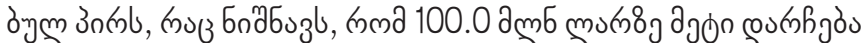
ззмбмanzol sa lodom man;

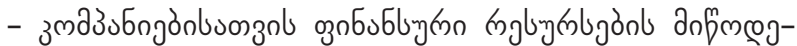

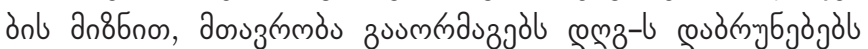

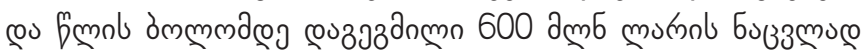

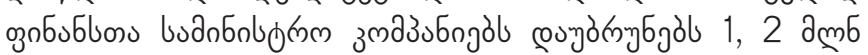

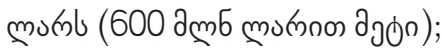

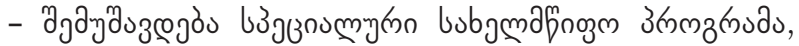

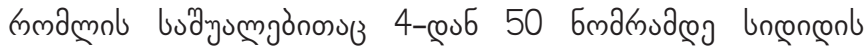

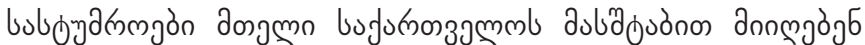

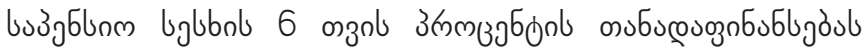

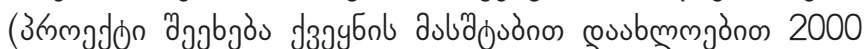

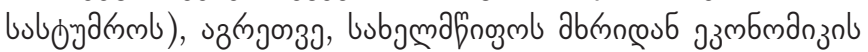

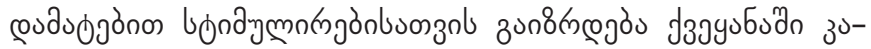

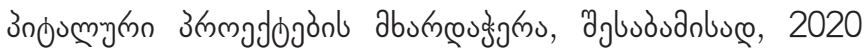

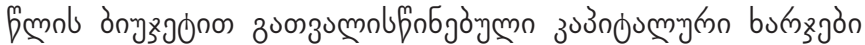

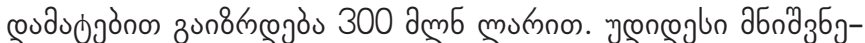

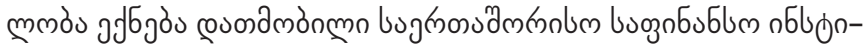

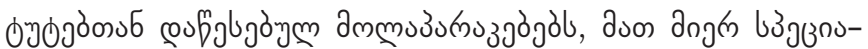

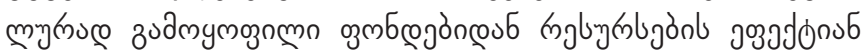

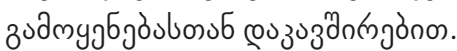

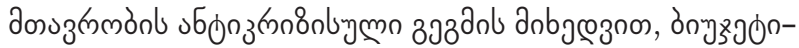

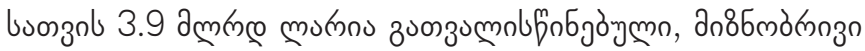

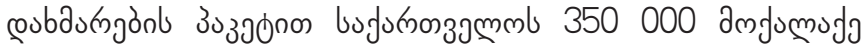
oburnzadmgab.

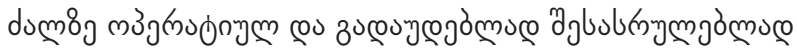

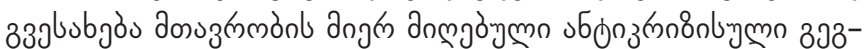

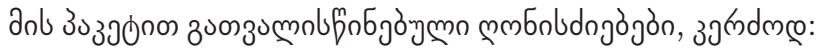

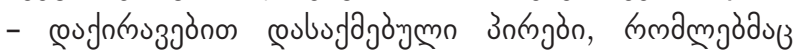

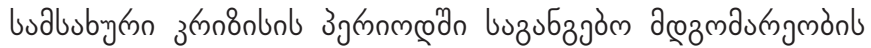

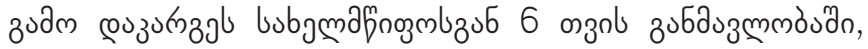

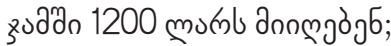

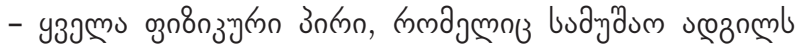

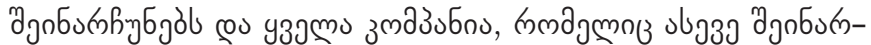

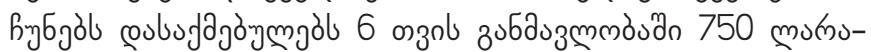
a

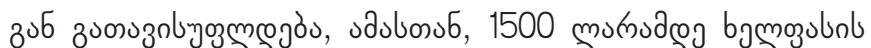

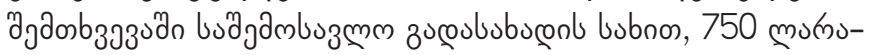

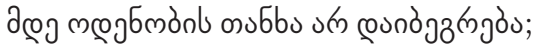

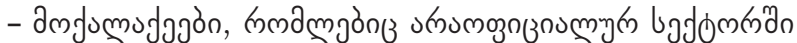

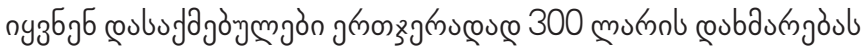
дnпмjòjb; 


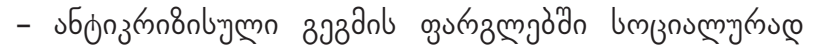

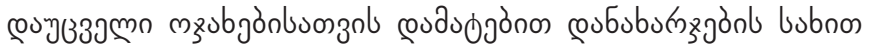

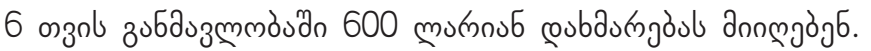

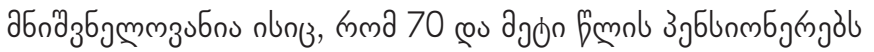

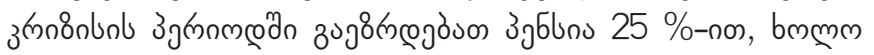

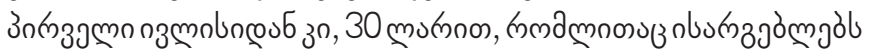

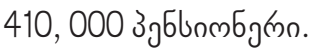

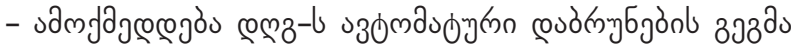

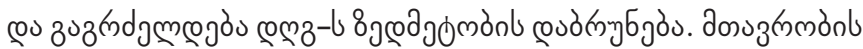

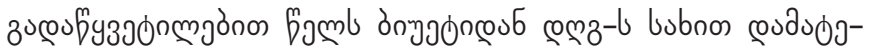

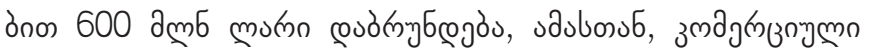

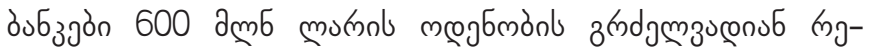

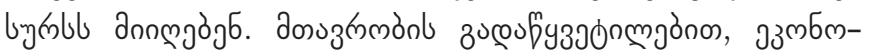

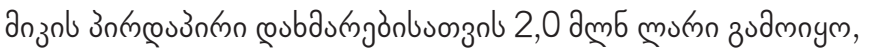

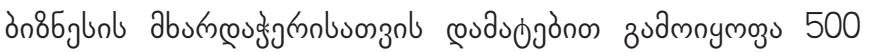

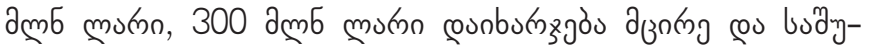

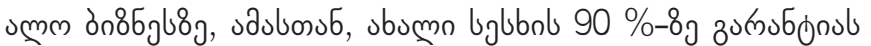

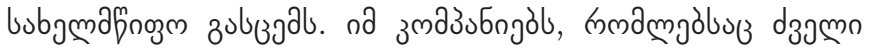

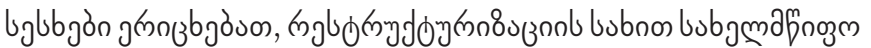

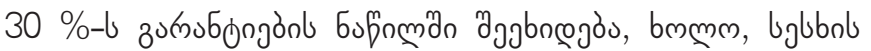

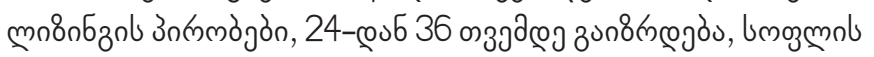

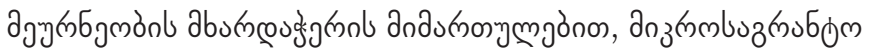

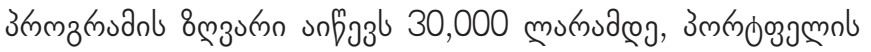

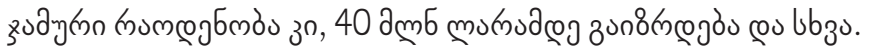

चб

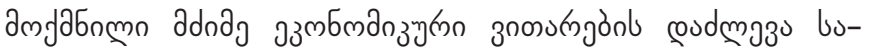

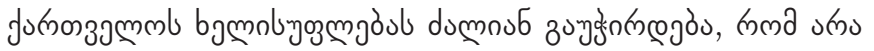

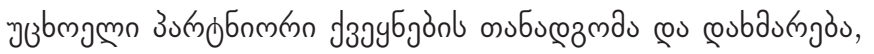

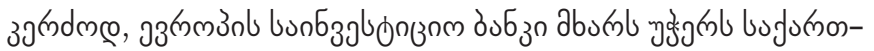

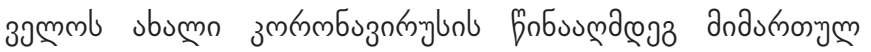

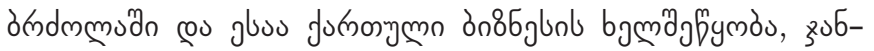

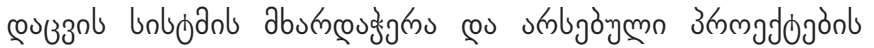
couffurngòymn zubbminzogmgòs.

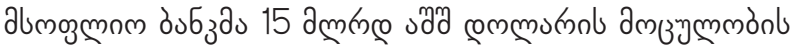

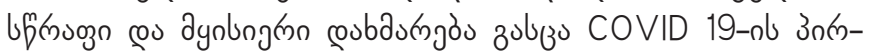
зэм зงдm

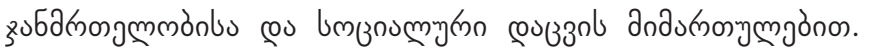

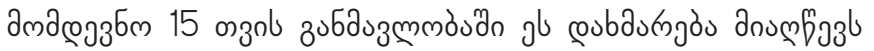

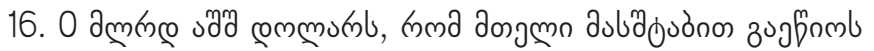

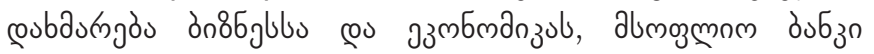

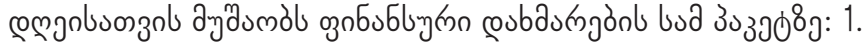

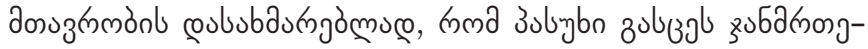

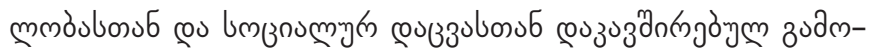

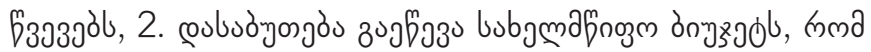

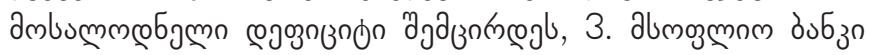

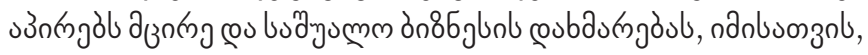

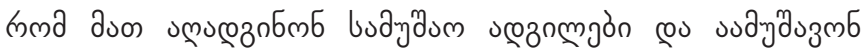

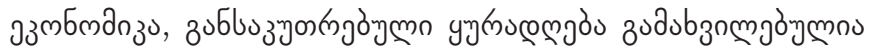

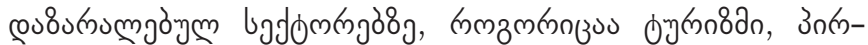
उगलm oुs

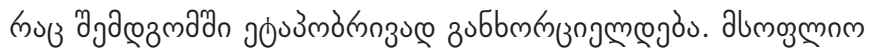

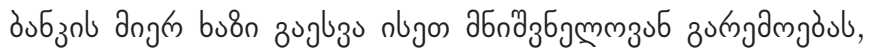

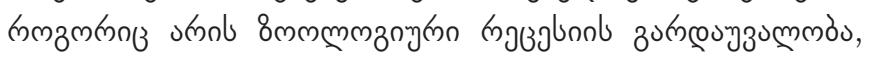

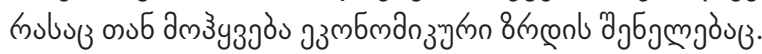

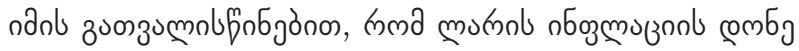

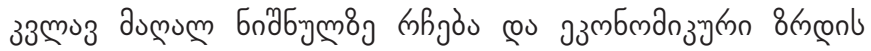

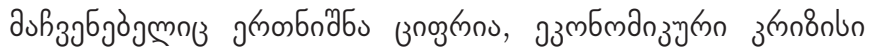

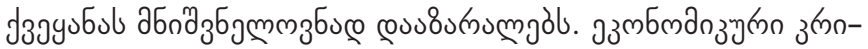

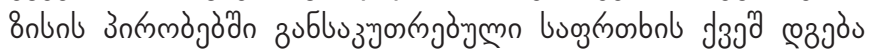

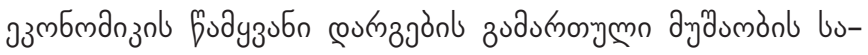

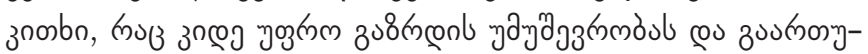

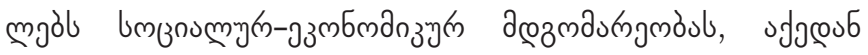

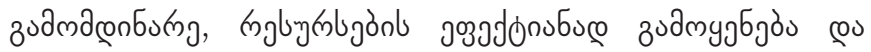

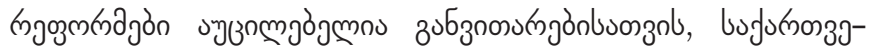

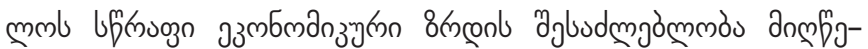

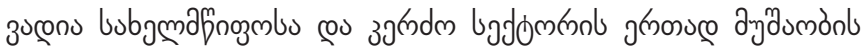

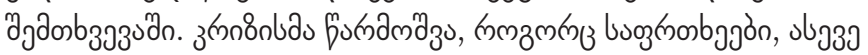

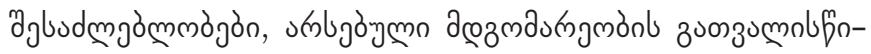

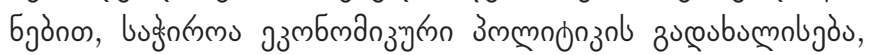

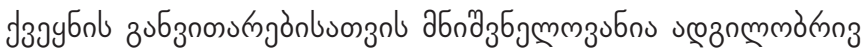

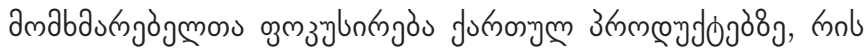

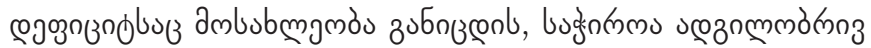

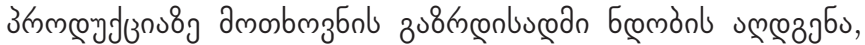

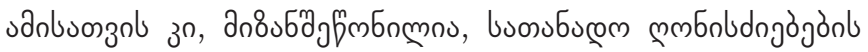

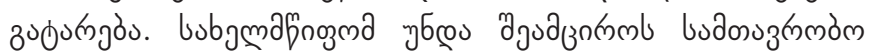

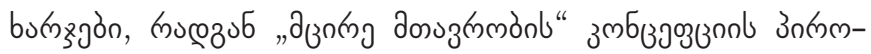

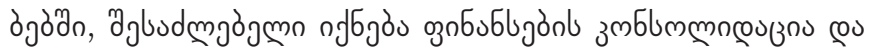

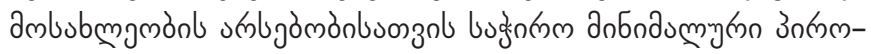

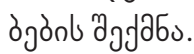




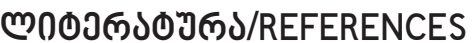

Ananiashvili, I., \& Papava, V., (2009). Taxes, demand and supply. Tbilisi: Publishing House -Siakhle. (In Georgian) Bakhtadze, L., Kakulia, R., \& Chikviladze, M., (2007). Tax case. Tbilisi. (In Georgian)

Kopaleishvili, T., \& Chikviladze, M. (2011). Taxes and taxation. Tbilisi. (In Georgian)

Jibuti, A., Kakulia, R., \& Bakhtadze, R. (2007). Public Finances. Tbilisi. (In Georgian)

Materials of the Ministry of Finance of Georgia, State Budget of Georgia for 2020. (In Georgian)

Tax Code of Georgia (2010). Tbilisi. (In Georgian)

Chikviladze, M. (2018). The Share in Percentage of Taxes in GDP. Ecoforum Journal, 7, 1 (14).

Atanelishvili, T., Chikviladze, M., \& Silagadze, N. (2017). About State Financial Control. Ecoforum Journal. 6, 1 (10).

Bedianashvili, G., Ivanov, Yu.B., Paientko, T. V. (2019). Tax Reforms in Ukraine and Georgia: Changing Priorities. Journal of Tax Reform. 5(2): 107-128.

Silagadze, A., Atanelishvili, T., \& Silagadze, N. (2019). Economic Doctrines: The Origins. Published by Nova Science Publishers, Inc. New York.

Silagadze, A., \& Zuniashvili, T. (2016). Georgia`s Economy against the Background of the Associate Agreement with the European Union. International Journal of Business and Management Studies, 59, 2: 533-540. (USA).

Zubiashvili, T., Chikviladze, M., \& Silagadze, N. (2018). Some Aspects of state External Debt. Ecoforum Journal 7 (2). 